\title{
Corpus
}

Archivos virtuales de la alteridad americana

Vol. 10, No. 1 | 2020

Enero / Junio 2020

\section{Desconexión y violencia en el Museo Policial}

\section{Martín Albornoz}

\section{OpenEdition}

\section{Journals}

Electronic version

URL: http://journals.openedition.org/corpusarchivos/3431

DOI: 10.4000/corpusarchivos.3431

ISSN: 1853-8037

\section{Publisher}

Diego Escolar

\section{Electronic reference}

Martín Albornoz, « Desconexión y violencia en el Museo Policial », Corpus [En línea], Vol. 10, №. 1 | 2020, Publicado el 28 junio 2020, consultado el 02 julio 2020. URL : http://journals.openedition.org/ corpusarchivos/3431; DOI : https://doi.org/10.4000/corpusarchivos.3431

This text was automatically generated on 2 July 2020.

Licencia Creative Commons: Atribución-NoComercial 2.5 Argentina (CC BY-NC 2.5 AR) 


\title{
Desconexión y violencia en el Museo Policial
}

\author{
Martín Albornoz
}

1 Al comenzar a escribir estas notas sobre el Museo Policial de la Policía Federal Argentina, no puedo evitar que venga a mi mente una fotografía originalmente publicada en Caras y Caretas que actualmente se conserva en el Archivo General de la Nación. Fue utilizada por el semanario ilustrado en julio de 1924, en una extensa crónica titulada: “¿Cómo se descubre un robo?”. ${ }^{1}$ En ella se lo ve al veterano y experimentado comisario Federico Foppiano, jefe de la sección Robos y Hurtos de la Comisaría de Investigaciones de la Policía de la Capital, contemplativo frente a una vitrina en la que pueden distinguirse una serie de llaves, ganzúas, puñales y un taladro incautados a los delincuentes de Buenos Aires gracias a la acción policial (ver Imagen 1). El conjunto formaba parte del Museo de la Policía de la Capital, en un momento muy preciso de su historia en el cual las piezas que lo componían estaban diseminadas en las oficinas de las distintas secciones que conformaban la Comisaría de Investigaciones: la mencionada Robos y Hurtos, Orden Social, Leyes especiales y Defraudaciones y Estafas. El Museo no estaba abierto al público y su pretensión no era tanto vincular ciertos objetos a un determinado pasado que se consideraba digno de cuidado y veneración, sino que, por el contrario, su función era contemporánea a las tareas que los agentes desplegaban día a día en la ciudad. Por decirlo de otro modo: los elementos e ingenios delictivos exhibidos estaban "vivos" e insertos en un presente que los dotaba de sentido. 


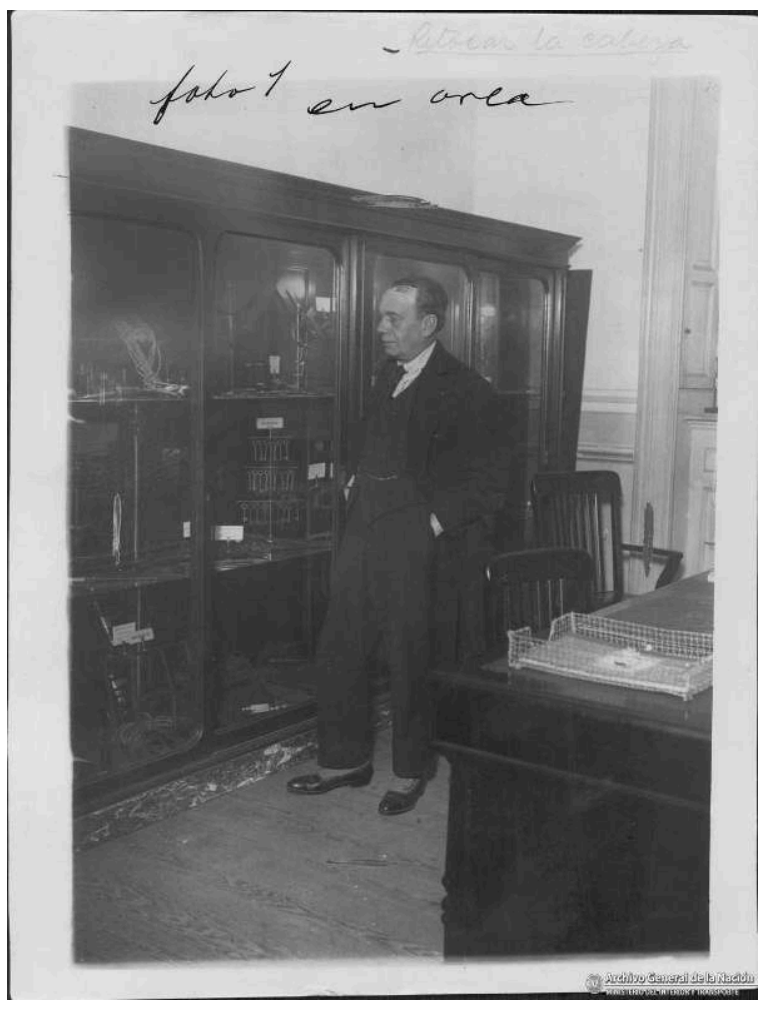

Imagen 1: "Federico Foppiano", Archivo General de la Nación, Departamento de Fotografía

El Museo había sido fundado en abril de 1899 con propósitos pedagógicos cuando la institucionalidad policial estaba en plena reconfiguración y las instancias formativas de los agentes eran escasas. En 1903, la colección se fragmentó, hasta que, en 1932, fue reagrupada en un mismo espacio museístico al que se le agregó el calificativo "histórico". El interés que los temas policiales y criminales generaban en la opinión pública -visibles en artículos periodísticos como el mencionado de Caras y Caretasparece haber pesado en la decisión. A su vez, se apostaba a que la dependencia, al convocar a un público profano amplio -atraído por las curiosidades y peculiaridades del mundo del crimen- propiciara cierto efecto preventivo. El comienzo de esta nueva etapa pública fue auspicioso. Las Memorias de Investigaciones de 1934 apuntaban que, solo en 1932, "casi 2500 personas han desfilado por las salas del museo y todas ellas han elogiado esta obra que viene a significar un adelanto para esta institución que por su organización y eficacia ha merecido siempre el aplauso y la admiración del pueblo". También se auguraba que el Museo iba a cobrar, en pocos años, "un lugar destacado dentro de nuestra institución". ${ }^{2} \mathrm{Al}$ año siguiente, lo habían visitado cerca de 7200 personas. ${ }^{3}$ Con el paso de las décadas, el Museo mudó varias veces de local. Originalmente situado en distintos espacios dentro del propio Departamento de Policía, en la década del cuarenta supo ocupar los altos de la Comisaría 19 hasta su traslado definitivo al lugar en el que hoy se encuentra, en pleno centro de la ciudad, en los pisos $7^{\circ}$ y $8^{\circ}$ del edificio de la Sede Social del Círculo de la Policía Federal.

3 En la actualidad, de forma casi obligada, la visita tiene que realizarse de la mano de una diligente policía que, ataviada con un delantal blanco, es la encargada de desplegar el argumento y el guión del Museo. El paso por las salas es veloz, las intervenciones de la guía, meramente descriptivas, sin grandes inflexiones emotivas, ni arrestos de solemnidad. En el $7^{\circ}$ piso, atravesamos la sala en la que se exponen, vistiendo unos 
antiguos maniquíes, uniformes policiales de distintas épocas. Luego un pequeño espacio que homenajea a los caídos en el cumplimiento del deber y, casi sin detenernos en el dedicado a las policías extranjeras y el destinado a las medallas y condecoraciones, nos demoramos en la sala que recupera elementos que refieren a las telecomunicaciones y las técnicas de investigación policial. Súbitamente, aflora un orgullo que es bastante fácil de comprender. Allí se ostentan verdaderos triunfos técnicos y se respira cierta atmósfera modernizante relacionada con pericias balísticas, la identificación dactiloscópica y la evolución de la comunicación policial. La manifiesta modernidad pasada de los complejos dispositivos tienen el anticlimático efecto de resaltar su arcaísmo.

4 Mi insistencia en observar cómo situaba el Museo a sus "otros" generó cierto desorden en la recorrida y nos obligó a un pequeño desvío. En respuesta a mi solicitud, subimos al octavo piso en el cual la narración y los objetos desplegados invierten el foco aunque el tono sigue oscilando entre la descripción y el anecdotario. Ya no se trata de ensalzar la pericia técnica y la potencia institucional de la policía, sino de iluminar las zonas oscuras del delito y sus prácticas. Con una organización que recuerda a las divisiones de la Comisaría de Investigaciones, el acento está puesto en diferentes modalidades delictivas. En las vitrinas y en las recreaciones se condensan y recrean el ingenio y el dinamismo de una pléyade de delincuentes. Desde timadores y curanderas, pasando por adivinas y anarquistas, hasta falsificadores de dinero, escruchantes, pistoleros y ladrones. Solo desentona, en todo el piso, el espacio dedicado a la fotografía policial, en el cual descansan una miríada de cámaras antiguas. La visita es igualmente rauda. Pude notar, lo que me resultó significativo, que la vitrina dedicada a Severino Di Giovanni, el célebre anarquista de acción que durante la década del veinte tuvo en vilo a la opinión pública porteña, no estaba situada en la sección dedicada al Orden Público, como yo hubiera pensado, sino a la dedicada a Robos y Hurtos.

De regreso al séptimo piso completamos la visita interrumpida por mi ansiedad. Solo restaba ver un gran salón en el cual se agrupan elementos vinculados con el cuerpo de bomberos $y$, en un costado, varias vitrinas que exponen cientos de armas de fuego utilizadas por la policía en el pasado. Enfrentada a esta, más grande y visible, hay otra vitrina que exhibe pistolas, fusiles y ametralladoras utilizadas por, según mi guía, "los malos". Retengo algunas fechas relacionadas con la historia reciente que acompañan los carteles descriptivos - que son absolutamente técnicos-, que me hacen pensar en los contextos históricos en que pudieron ser utilizadas.

6 La primera sensación que trasmite el Museo es la de un fuerte anacronismo. El horizonte de expectativas que marcó su origen está eclipsado o reconfigurado. El anhelo pedagógico y preventivo parece extinto y se hace muy difícil pensar que en la actual formación de los futuros agentes policiales esté incluido obligatoriamente el estudio de la colección. También cuesta imaginar que esas vitrinas atiborradas de objetos mínima o nulamente referenciados pueda ser razón de alguna clase de orgullo público manifiesto. La conexión con las actividades policiales del presente es, por lo menos, difusa. Finalmente, consultando el libro de visitas, no hay que hacer muchas cuentas para darse cuenta de que hoy en día, ni por asomo, lo transitan siete mil curiosos. El presente brilla por su ausencia. Solo, respondiendo más a una lógica de depósito que a una lógica museística, la enorme y esmerada maqueta utilizada en la reconstrucción a escala del atentado a la AMIA contraría esa certeza. El cartel que la describe tiene inesperadas resonancias de actualidad: "desde el año de la ocurrencia 
hasta la actualidad, la tarea de la justicia ha pasado por avatares que son del dominio público, durante las cuales se ha agregado la muerte aún no aclarada del fiscal de la causa, Alberto Nisman, en 2015".

7 Sin embargo, el Museo existe y, además de una historia institucional, tiene otras historias que narrar, que efectivamente se narran, a partir de cierta curaduría que por momentos parece tan antigua como las huellas materiales que la sostienen. Una de esas historias, particularmente sensible, es la que se relaciona con los ejercicios de la violencia y los modos de procesarla por parte de la propia policía a partir de un artefacto cultural específico como lo es el museo, en el cual los silencios y omisiones, los pequeños desvíos e incluso las distorsiones cuentan tanto como lo que explícitamente se muestra.

8 Pensando puntualmente en lo relativo a la violencia estatal, completamente ubicua en todos los objetos que componen el Museo, hay que decir que en la curaduría, al menos, está obliterada. Hasta qué punto esto es deliberado es difícil saberlo. Esto sucede, en parte, por la propia organización temática que divide a ambos pisos. Los policías en uno, los transgresores y enemigos de la ley en el otro. En el primero, por ejemplo, una estantería con puertas de vidrio muestra, sin más, cartuchos de gases lacrimógenos y vomitivos, máscaras antigás y pistolas lanza gases. Se puede asumir que todo esto es antiguo, pero nada se dice sobre su contexto pasado o sobre los problemas a los que buscaba dar respuesta. Su clara vinculación directa con el orden público hace más flagrante la omisión. En el otro piso, un surtido conjunto de dagas descansa sin mayores referencias a las situaciones en las que fueron incautadas. La insólita y extemporánea sala dedicada a la "toxicomanía" está complementada con unas sombrías fotos en blanco y negro de jóvenes cuyas únicas explicaciones descansan en citas entresacadas de algún informe pretérito de la Organización Mundial de la Salud. Los elementos relacionados con el tráfico y consumo de drogas no tienen una sola mención al tipo de tareas que la policía desplegó (y despliega) contra el narcotráfico. Pocas veces aflora el intercambio entre ambos mundos.

En esta negación de la interacción se alisa el relato y se atenúa su dimensión violenta. La forma en la que el acervo es mostrado, obedeciendo, al parecer, al criterio de la mera exposición, favorece esa impresión. Un arma en su propia materialidad, entre otras cien, poco nos dice en sí misma sobre la decisión que pudo haber mediado en su utilización. De esta forma, los objetos de agresión, siguiendo a Mariana Sirimarco, se esterilizan y "el éxito de esta operación sanitizante" reposa en el mecanismo de "hacer que el trabajo sobre la memoria sobre el tiempo no opere rememorando sino re/des/ conectando" (2019, p. 22). Los signos de la violencia ejercida se diluyen en el arrumbamiento y el aislamiento de esa acumulación.

10 Algo parecido sucede con la violencia recibida. En el espacio conmemorativo destinado a los "caídos en el cumplimiento del deber", las circunstancias en las cuales a un policía le ha tocado perder la vida está ausente: "silbato que perteneció a un agente muerto durante la Revolución de 1890". Nada más. Un enorme cuadro de Juan Alberto Lartigau, asesinado el 14 de noviembre de 1909, junto al entonces jefe de la Policía de la Capital, Ramón Falcón, por el anarquista ruso Simón Radowitzky, es un testimonio mudo de su propia historia. Como emergencia de una evocación emotiva, resultan más ensalzados aquellos que por obra de la suerte sobreviven, -por ejemplo gracias a una oportuna bala disparada por un delincuente que va a parar al nudo de una corbata o de lleno a la placa que portaba un agente- que los propios muertos (Sirimarco, 2014). 
11 El caso de Ramón Falcón es interesante por la vaporosidad de su presencia. Las circunstancias de su muerte violenta, su severo perfil militar y represivo, que en las lecturas más aventuradas presagian dramas futuros, y su rol destacado con las reformas autoritarias que tuvieron lugar en la policía en los años previos a las celebraciones del centenario de la Revolución de Mayo de 1810, hicieron que los modos en los que se construyó su memoria estuvieran atados a los vaivenes políticos de la historia argentina a lo largo del siglo XX. Desde la entronización inmediata como mártir policial, pasando por su consolidación simbólica en la década del treinta, hasta su reactivación negativa en los años posteriores a la última dictadura. ${ }^{4}$ Los rastros de esas pujas de sentido, en principio, no son mencionados, y los elementos que permitirían su evocación en el Museo están desperdigados: el escritorio que utilizó hasta su último día y una pequeña estatua. Surge la inevitable pregunta de si esto fue siempre así. Consultando un artículo generosamente ilustrado que publicó la revista Mundo Policial en 1989, observo que una fotografía muestra una puesta en escena que ya no está más. En ella, custodiado por dos maniquíes prolijamente uniformados, un busto de Ramón Falcón. ${ }^{5}$ Algo más específica resulta la narración de su trayectoria en unas hojas que están en una especie de cuaderno de metal. No hay nada polémico ni reivindicativo en ellas y su presencia en el Museo no organiza ninguna invocación privilegiada. Su nombre es uno más junto al de otros jefes policiales.

12 Si se toma como problema la violencia política, específicamente la policial, para el que ha visitado otros museos similares, recorrer el Museo Policial de la Policía Federal Argentina es una experiencia desconcertante. En París, por ejemplo, el Museo de la Prefectura se sostiene en una narración orgullosa de su historia y su accionar en diferentes dramas políticos, que van desde la Comuna de París en 1871, pasando por las bombas no estalladas de los anarquistas, hasta el Mayo Francés. En Buenos Aires, de acuerdo con lo ya dicho, la atenuación de la violencia explícita genera un efecto disruptivo. Sin embargo, ese ocultamiento tiene una extraña virtud: la mezcla. La cantidad de escenarios en los que a la policía le tocó actuar, por momentos, hacen artificial esa división. La trayectoria del mencionado Federico Foppiano dentro de la Comisaría de Investigaciones es ilustrativa. Jefe de la División Orden Social en la primera década del siglo XX, se jubiló luego de casi tres décadas de actuación como jefe de Robos y Hurtos. De este modo, las líneas que separan, dentro del know-how policial, lo político de lo social, son porosas. ${ }^{6}$ Creo que la inclusión del anarquista Severino Di Giovanni en la sección Robos y Hurtos, que tanto llamó mi atención en un primer momento, resulta productiva.

13 La vitrina dedicada a su figura tiene claramente el propósito de realzar su condición de trofeo. Está ubicada en una sala que lleva el nombre del que quizás fue el último miembro de Investigaciones de renombre público: el comisario Evaristo Meneses. Con fama de policía duro e incorruptible, Meneses en su lucha contra la delincuencia organizada no quedó eximido de denuncias por lo que en otro tiempo se llamaban apremios ilegales. ${ }^{7}$ Volviendo a Di Giovanni, su paso por el mundo criminal es evocado a partir de sus revólveres, las patentes de coches falsas que utilizaba en sus huidas, las esposas que tenía en el momento de ser fusilado. Muy a tono con el guión del museo, nada se dice sobre sus acciones, ni sobre las ideas que las sustentaban. Tampoco sobre sus víctimas, entre las que se contaban algunos policías. Sin embargo, se expone un ejemplar del diario La Nación del día de su detención, en cuyo titular principal puede 
leerse: “Tras una trágica persecución el asaltante Severino Di Giovanni cayó ayer en poder de la policía".

Al Museo Policial de la Policía Federal le toca intervenir en una zona ríspida de la propia historia de la institución: las relaciones siempre tensas entre policía y pueblo. Lila Caimari observó que ese vínculo estuvo marcado por cierta "eufemización" de la violencia, real o potencial, que los policías desplegaban en su tarea (2012, p. 188). A diferencia de otros soportes conmemorativos, como memorias o relatos institucionales, en el Museo esa "eufemización" resulta más patente, en la medida en que gran parte de su acervo remite a distintas formas, más o menos intensas, de violencia. Las descontextualizaciones, omisiones o desfiguraciones que lo caracterizan parecieran jugar en ese sentido.

\section{BIBLIOGRAPHY}

Albornoz, M. y Galeano, D. (en prensa). A History of the City of Buenos Aires Police Bureau of Investigations, 1880-1910. En A. Carrizo y M. Riekenberg (Eds.), Doing cop culture. Beiträge zur Geschichte der modernen Polizie in Lateinamerika. Leipzig: Universität Leipzig.

Caimari, L. (2012). Mientras la ciudad duerme. Pistoleros, policías y periodistas en Buenos Aires, 1920-1945. Buenos Aires: Siglo XXI.

García Ferrari, M. y Gayol, S. (2015). Ramón Falcón: asesinato y usos políticos de la muerte. En S. Gayol y G. Kessler (Eds.), Muerte, política y sociedad en Argentina, (pp. 61-83). Buenos Aires: Edhasa.

Sirimarco, M. (2014). La cosa y la palabra. Relato y emocionalidad en un museo policial. Revista del Museo de Antropología, 7(1), 177-188.

Sirimarco, M. (2019). Presentación. Museos y fuerzas de seguridad en la Argentina. En M.

Sirimarco (coord.), Narrar el oficio. Los museos de las fuerzas de seguridad como espacio de ficciones fundadoras, (pp, 9-30), Buenos Aires: Biblos.

\section{NOTES}

1. “Cómo se descubre un robo?”, Caras y Caretas, 19 de julio de 1924, Año XXVI, N¹346, p.152.

2. Policía de la Capital Federal, Memoria de Investigaciones. Años 1932-1933, Buenos Aires, 1937, p. 130.

3. Policía de la Capital Federal, Memoria de Investigaciones. Años 1932-1933, op. cit., p.343.

4. Ver García Ferrari y Gayol (2015).

5. Museo Policial. Policía Federal Argentina, Boletín informativo - Anexo Mundo Policial, №61, 1989.

6. Recientemente, con Diego Galeano, dedicamos un trabajo a estudiar específicamente las primeras décadas de existencia de la Comisaría de Investigaciones. Contra lo que la historiografía había sencillamente mencionado, las tareas de esa dependencia distaban de tener como único objetivo la defensa contra el anarquismo u otras expresiones de disidencia política. Dentro de su enorme rango de actuación, entraba también la vigilancia y prevención de una variada gama de delitos. Al respecto, ver Albornoz y Galeano (en prensa). 
7. Sobre Meneses, recomiendo ver la película documental Evaristo de Mariano Petrecca, realizada en 2016.

\section{AUTHOR}

\section{MARTÍN ALBORNOZ}

Universidad Nacional de San Martín, Instituto de Altos Estudios Sociales y Consejo Nacional de Investigaciones Científicas y Técnicas, Argentina.

Correo electrónico: martinalbornozc@gmail.com 